\title{
AROUND 3D BOLTZMANN NON LINEAR OPERATOR WITHOUT ANGULAR CUTOFF, A NEW FORMULATION
}

\author{
RADJESVARAne AleXANDRE ${ }^{1}$
}

\begin{abstract}
We propose a new formulation of the 3D Boltzmann non linear operator, without assuming Grad's angular cutoff hypothesis, and for intermolecular laws behaving as $1 / r^{s}$, with $s>2$. It involves natural pseudo differential operators, under a form which is analogous to the Landau operator. It may be used in the study of the associated equations, and more precisely in the non homogeneous framework.
\end{abstract}

Mathematics Subject Classification. 35D05, 35D10, 35K70, 82B40.

Received: June 10, 1999. Revised: September 22 and October 22, 1999.

\section{INTRODUCTION}

In this paper, we introduce a Pseudo-Differential approach of the Boltzmann 3D non linear operator, when one does not assume Grad's angular cutoff hypothesis.

For functions $f(v), v \in \mathbb{R}^{3}$, this operator has the following form

$$
Q(f, f)(v) \equiv \int_{\mathbb{R}_{v_{1}}^{3}} \int_{S_{\omega}^{2}} \mathrm{~d} v_{1} \mathrm{~d} \omega\left\{f\left(v^{\prime}\right) f\left(v_{1}^{\prime}\right)-f(v) f\left(v_{1}\right)\right\} B\left(\left|v-v_{1}\right|,\left|\left(\frac{v-v_{1}}{\left|v-v_{1}\right|}, \omega\right)\right|\right)
$$

Above $v, v_{1} \in \mathbb{R}^{3}$, and $v^{\prime}=v+\left(v_{1}-v, \omega\right) \omega, v_{1}^{\prime}=v_{1}-\left(v_{1}-v, \omega\right) \omega$ denote the so-called post collisional velocities, for a given $\omega \in S^{2}$, unit sphere of $\mathbb{R}^{3}$.

As indicated, the collisional kernel $B(.,$.$) is a given function of the relative velocity \left|v-v_{1}\right|$ and on the angle $\left|\left(\frac{v-v_{1}}{\left|v-v_{1}\right|}, \omega\right)\right|$.

For the precise physical framework, we refer to $[14,15]$.

In most of the mathematical works concerned with the associated homogeneous and non homogeneous equations, one assumes the angular cutoff hypothesis of H. Grad. Roughly, this means that

$$
\omega \rightarrow B\left(\left|v-v_{1}\right|,\left|\left(\frac{v-v_{1}}{\left|v-v_{1}\right|}, \omega\right)\right|\right) \in L^{1}\left(S^{2}\right)
$$

For instance, one can consult the papers [21, 22, 24, 25,33].

Keywords and phrases. Boltzmann operator, ellipticity, non angular cutoff, pseudo differential operators.

1 MAPMO, UMR 6627, Département de Mathématiques, Université d'Orléans, BP 675945067 Orleans Cedex 2, France. e-mail: alexandr@labomath.univ-orleans.fr 
On the other hand, it is well known since the outset of the mathematical studies on the Boltzmann equation (actually this has led Grad to his cutoff hypothesis), that (1.2) is almost never satisfied for real physical interactions.

Indeed, see $[14,33]$, in 3 dimensions, $B$ has the following typical form

$$
B\left(\left|v-v_{1}\right|,\left|\left(\frac{v-v_{1}}{\left|v-v_{1}\right|}, \omega\right)\right|\right) \equiv\left|v-v_{1}\right|^{\gamma} \frac{1}{\left|\left(\frac{v-v_{1}}{\left|v-v_{1}\right|}, \omega\right)\right|^{\nu}}
$$

which is a cross section close to that given by forces in $\frac{1}{r^{s}}$, with $s>2$. The parameters $\gamma$ and $\nu$ are given by

$$
\gamma=\gamma(s)=\frac{s-5}{s-1}, \nu=\nu(s)=\frac{s+1}{s-1}
$$

For such values, one checks that (1.2) fails to hold, and this is called the non cutoff case. It explains one of the main difficulties in giving a meaning to $Q(f, f)(v)$ in (1.1), for non smooth $f$, as the usual splitting of $Q$ into the gain and loss terms is meaningless.

There are only few papers concerned with that case.

For the homogeneous equation, that is without dependence in the variable $x$ (the position), Arkeryd [10,11] has dealt with the existence, when entropic bounds are assumed on the initial data. His work was extended recently by $[23,30]$.

Note that such solutions are built with few informations, that is only moments and entropic estimates are known.

In the 2D homogeneous and non cutoff case, more informations are available, and in particuliar, regularity results have been proven to hold by Desvillettes [17-19], see also [20].

Let us also mention the paper of Pao [27] about the linearized Boltzmann operator again in the non cutoff case.

We recall the work of Ukai [33] on the non homogeneous equation in the non cutoff case, where he produced local (in time) solutions in the Gevray class.

We would like also to mention the related papers $[4,8,26,31]$ showing regularity estimates from the entropic dissipation rate.

As a final remark, we point out the paper [9], where not only assumption (1.3) is improved, but also produces weak solutions without the way introduced herein.

In this paper, we propose a new formulation of $Q$ in the non cutoff case. Previous formulations exist already, see for instance [32], but our strategy will be different here. Indeed, our formulation introduces a pseudodifferential approach to $Q$, and as such, is more adapted to a functional treatment of the Boltzmann equation.

This way has been settled up in $[1,2]$ in the $3 \mathrm{D}$ linear context, where we gave an analysis of the structure of the associated (non) homogeneous equation, exhibiting new features, and among these ones, the so-called irreversibility, or what seems to be one mathematical traduction of this physical notion.

The present paper is concerned with the non linear situation, that is for the operator $Q$ as given by (1.1), with the assumptions (1.3) and (1.4), and some generalisations.

In Section 2, we display this decomposition of $Q$. It is given by Theorem 2.1 and shows clearly the difference between the cutoff and non cutoff cases. In particular, in the non cut off case, the operator $Q$ acts on $f$ as a pseudo-differential operator of strictly positive order, but with an exotic positive symbol. This fact was already noticed by Pao in the linearised setting [27], but using older tools from pdo theory.

Let us mention that we shall not discuss the usual questions, such as existence, regularity or even asymptotics of the full non homogeneous Boltzmann equation, in the non cutoff case. But the result (or method) presented here is used in at least two papers (and already in [1,2]): in [5], where we produce renormalised solutions and in [7] where global solutions are build for some small initial data. Also, an other important application for the linearised operator is actually worked out [6]. 
The final Section 3 is concerned with the so-called Boltzmann-Coulomb case, an important cross section occurring for instance in plasma physics $[12,14,16]$. As such, it stands outside the non cutoff case, but we show that the method of Section 2. applies for this collisional kernel.

Some results of this paper have been announced in [3].

\section{Decomposition of $Q$} (1.1).

We give here a pseudo-differential decomposition of the 3D Boltzmann non linear operator $Q$ as given by

For practical purposes, we consider the associated quadratic bilinear operator (not symmetric) which writes for $f=f(v), g=g(v)$, smooth functions of $v \in \mathbb{R}^{3}$

$$
Q(f, g)(v) \equiv \int_{\mathbb{R}_{v_{1}}^{3}} \int_{S_{\omega}^{2}} \mathrm{~d} v_{1} \mathrm{~d} \omega\left\{f\left(v^{\prime}\right) g\left(v_{1}^{\prime}\right)-f(v) g\left(v_{1}\right)\right\} B\left(\left|v-v_{1}\right|,\left|\left(\frac{v-v_{1}}{\left|v-v_{1}\right|}, \omega\right)\right|\right)
$$

where the notations are those of Section 1.

We recall that the collision kernel $B$ is given as

$$
B\left(\left|v-v_{1}\right|,\left|\left(\frac{v-v_{1}}{\left|v-v_{1}\right|}, \omega\right)\right|\right) \equiv\left|v-v_{1}\right|^{\gamma} \frac{1}{\left|\left(\frac{v-v_{1}}{\left|v-v_{1}\right|}, \omega\right)\right|^{\nu}},
$$

and the critical parameters are defined by

$$
\gamma=\gamma(s)=\frac{s-5}{s-1}, \nu=\nu(s)=\frac{s+1}{s-1}
$$

and we always assume $s>2$.

The formulation of $Q$ announced in the Introduction is given by

Theorem 2.1. Let $Q$ be the Boltzmann 3D non linear operator given by (2.1), with B, $\gamma$ and $\nu$ given by (2.2a) and (2.3). Then, one has the decomposition

$$
\begin{aligned}
Q(f, g)(v)= & -C_{s}^{\prime} \int_{\mathbb{R}_{\alpha}^{3}} \mathrm{~d} \alpha g(\alpha+v)|\alpha|^{\gamma+\nu}|S(\alpha) \cdot D|^{\nu-1}(f)(v) \\
& +C_{s}^{\prime} \cdot f(v) \cdot \int_{\mathbb{R}_{\alpha}^{3}} \mathrm{~d} \alpha|\alpha|^{\gamma+\nu}|S(\alpha) \cdot D|^{\nu-1}(g)(\alpha+v) \\
& +\int_{R_{v_{1}}^{3}} \int_{S_{w}^{2}} \mathrm{~d} v_{1} \mathrm{~d} w\left\{f^{\prime} f_{1}^{\prime}-f f_{1}\right\} B^{c}\left(\left|v-v_{1}\right|,\left|\left(\frac{v-v_{1}}{\left|v-v_{1}\right|}, \omega\right)\right|\right) .
\end{aligned}
$$

Above, we used standard pdo notations. $C_{s}^{\prime}$ is a fixed constant depending only on s, and given through (2.19) and (2.23). $S(\alpha)$ denotes the orthogonal projection operator over $E_{0, \alpha}$, the hyperplane through 0 and orthogonal to $\alpha$, and

$$
B^{c}\left(\left|v-v_{1}\right|,\left|\left(\frac{v-v_{1}}{\left|v-v_{1}\right|}, \omega\right)\right|\right) \equiv \frac{1}{\left|v^{\prime}-v\right|^{\nu}}\left[\left\{\left|v_{1}-v^{\prime}\right|^{2}+\left|v^{\prime}-v\right|^{2}\right\}^{\frac{\gamma+\nu}{2}}-\left|v_{1}-v^{\prime}\right|^{\gamma+\nu}\right] .
$$

One has $B^{c} \geq 0, B^{c} \in L_{l o c}^{1}\left(\mathbb{R}^{3} \times S^{2}\right)$, and more precisely

$$
B^{c}(., .) \leq C\left|v_{1}-v\right|^{\gamma} \frac{1}{\left\{1-\left|\left(\frac{v-v_{1}}{\left|v-v_{1}\right|}, \omega\right)\right|^{2}\right\}^{1-\frac{\gamma+\nu}{2}}} \cdot \frac{1}{\left|\left(\frac{v-v_{1}}{\left|v-v_{1}\right|}, \omega\right)\right|^{\nu-2}} .
$$


Remark 2.1. Note that the last term behaves exactly like a Boltzmann operator with cutoff, cf. the papers mentioned in Section 1.

Remark 2.2. Let us comment on the first term of the decomposition given by Theorem 2.1. There are at least two ways to consider this term. One may integrate in $\alpha$ first, then in $\xi$, or reverse the integrations. In the first way, setting

$$
l(v, \xi)=\int_{\alpha} \mathrm{d} \alpha|\alpha|^{\gamma+\nu} g(\alpha+v)|S(\alpha) \xi|^{\nu-1},
$$

$l$ is clearly homogeneous with respect to the variable $\xi$. Furthermore, if we assume $g \geq 0,\left(1+|v|^{2}\right) g \in L^{1}$, then $l$ is well defined, and one has a bound as

$$
0 \leq l(v, \xi) \leq C\left(\left\|\left(1+|\alpha|^{2}\right) g\right\|_{L^{1}}\right)\left(1+|v|^{2}\right)|\xi|^{\nu-1} .
$$

The bad point is the lack of control on derivatives of $l$, and thus the standard pdo theory does not apply.

In the second way, we fix $\alpha$, and introduce a cutoff around the singularity $|S(\alpha) \xi|$, that is a function $\phi$, smooth and such that

$$
\phi: \mathbb{R}^{+} \rightarrow[0,1],=0 \text { for } t \geq 1,=1 \text { for } t \leq 1 / 2 .
$$

Then, we split $|S(\alpha) \xi|^{\nu-1}$ as

$$
|S(\alpha) \xi|^{\nu-1}=|S(\alpha) \cdot \xi|^{\nu-1} \phi(|S(\alpha) \cdot \xi|)+|S(\alpha) \cdot \xi|^{\nu-1}\{1-\phi(|S(\alpha) \cdot \xi|)\} .
$$

Now, the first term is bounded, while the second one has no singularities, for fixed $\alpha$, and is still homogeneous for large $|S(\alpha) \xi|$.

In fact, we can say more. From

$$
|S(\alpha) \cdot D|^{\nu-1} \phi(|S(\alpha) \cdot D|)(f)(v)=\int_{\mathbb{R}_{\xi}^{3}} \mathrm{~d} \xi|S(\alpha) \cdot \xi|^{\nu-1} \phi(|S(\alpha) \cdot \xi|) \hat{f}(\xi) e^{i \xi \cdot v},
$$

let us consider a basis of $\mathbb{R}^{3}$, with first vector $\frac{\alpha}{|\alpha|}$, and express $\xi$ and $v$ in these coordinates. Then the term above writes as

$$
\int_{\mathbb{R}^{2}} \mathrm{~d} \xi_{2} \mathrm{~d} \xi_{3}\left|\xi_{2}^{2}+\xi_{3}^{2}\right|^{\frac{\nu-1}{2}} \phi\left(\left|\xi_{2}^{2}+\xi_{3}^{2}\right|^{\frac{1}{2}}\right) \hat{f}^{2,3}\left(v_{1}, \xi_{2}, \xi_{3}\right) e^{i\left(\xi_{2} v_{2}+\xi_{3} v_{3}\right)}
$$

where $\hat{f}^{2,3}$ denotes Fourier transform with respect to the last two variables. Now, as $\phi$ has compact support, we get that $|S(\alpha) \cdot D|^{\nu-1} \phi(|S(\alpha) . D|)(f)(v)$ is well defined.

Furthermore, we see that $|S(\alpha) \cdot \xi|^{\nu-1}\{1-\phi(|S(\alpha) . \xi|)\}$ is a good symbol, as in particular it is supported outside the set $|S(\alpha) \cdot \xi| \geq 1 / 2$. But this method is difficult to apply in the non linear setting of the Boltzmann equation, when assuming the usual entropic bounds.

One full correct way is explained in [5] and makes a deep use of results from [28,29] and references therein, producing renormalised solutions for the Boltzmann non homogeneous equation. Other applications of Theorem 2.1 (or method of proof) are also given in the linear (or linearised) context, see for instance $[1,2,6]$.

Remark 2.3. One may allow other kernels $B$. We just mention the following one, which is used in $[5,7]$, as it leads to the easiest mathematical analysis. Assume that we take

$$
B(., .)=\chi\left(\left|v_{1}-v^{\prime}\right|\right) \frac{1}{\left|v-v^{\prime}\right|}
$$


with the same exponent $\nu$ and where $\chi$ is a positive (say smooth) function. Then the method of proof of Theorem 2.1 applies again, and in that case, we get

$$
\begin{aligned}
Q(f, g)(v)=-C_{s}^{\prime} \int_{\mathbb{R}_{\alpha}^{3}} \mathrm{~d} \alpha g(\alpha+v) \chi(|\alpha|)|\alpha|^{\nu} \mid & \left.S(\alpha) \cdot D\right|^{\nu-1}(f)(v) \\
& +C_{s}^{\prime} f(v) \int_{\mathbb{R}_{\alpha}^{3}} \mathrm{~d} \alpha \chi(|\alpha|)|\alpha|^{\nu}|S(\alpha) \cdot D|^{\nu-1}(g)(\alpha+v) .
\end{aligned}
$$

Note that we have only two terms compared with the previous assumption (2.2a).

Remark 2.4. On the formulation given by Theorem 2.1, the link with the Landau operator, see [30-32] for instance, is more clear.

The rest of the section is devoted to the proof of Theorem 2.1.

Proof. (of Theorem 2.1).

Firstly, since $v^{\prime}=v+\left(v_{1}-v, \omega\right) \omega$, we have $\frac{v^{\prime}-v}{\left|v_{1}-v\right|}=\left(\frac{v_{1}-v}{\left|v_{1}-v\right|}, \omega\right) \omega$. In particular, one has the relation

$$
\left|\left(\frac{v_{1}-v}{\left|v_{1}-v\right|}, \omega\right)\right|=\frac{\left|v^{\prime}-v\right|}{\left|v_{1}-v\right|}
$$

It follows that $\frac{1}{\left|\left(\frac{v-v_{1}}{\left|v-v_{1}\right|}, \omega\right)\right|^{\nu}}=\frac{\left|v-v_{1}\right|^{\nu}}{\left|v^{\prime}-v\right|^{\nu}}$ and

$$
Q(f, g)(v) \equiv \int_{\mathbb{R}_{v_{1}}^{3}} \int_{S_{\omega}^{2}}\left\{f\left(v^{\prime}\right) g\left(v_{1}^{\prime}\right)-f(v) g\left(v_{1}\right)\right\}\left|v-v_{1}\right|^{\gamma+\nu} \frac{\mathrm{d} v_{1} \mathrm{~d} \omega}{\left|v^{\prime}-v\right|^{\nu}} .
$$

Note that $\gamma+\nu=\frac{2(s-2)}{s-1}>0$ as $s>2$. Next, we write $(2.6)$ as

$$
Q(f, g)(v) \equiv \int_{\mathbb{R}_{v_{1}}^{3}} \int_{S_{\omega}^{2}}\left\{f\left(v^{\prime}\right) g\left(v_{1}^{\prime}\right)-f(v) g\left(v_{1}\right)\right\}\left|\left(v_{1}-v^{\prime}\right)+\left(v^{\prime}-v\right)\right|^{\gamma+\nu} \frac{\mathrm{d} v_{1} \mathrm{~d} \omega}{\left|v^{\prime}-v\right|^{\nu}} .
$$

Noticing that $\left(v_{1}-v^{\prime}\right) \cdot\left(v^{\prime}-v\right)=0$, we deduce that

$$
Q(f, g)(v) \equiv \int_{\mathbb{R}_{v_{1}}^{3}} \int_{S_{\omega}^{2}}\left\{f\left(v^{\prime}\right) g\left(v_{1}^{\prime}\right)-f(v) g\left(v_{1}\right)\right\}\left\{\left|v_{1}-v^{\prime}\right|^{2}+\left|v^{\prime}-v\right|^{2}\right\}^{\frac{\gamma+\nu}{2}} \frac{\mathrm{d} v_{1} \mathrm{~d} \omega}{\left|v^{\prime}-v\right|^{\nu}}
$$

that we split as

$$
Q(f, g)(v)=Q_{1}(f, g)(v)+Q_{2}(f, g)(v)
$$

with

$$
Q_{1}(f, g)(v) \equiv \int_{\mathbb{R}_{v_{1}}^{3}} \int_{S_{\omega}^{2}}\left\{f\left(v^{\prime}\right) g\left(v_{1}^{\prime}\right)-f(v) g\left(v_{1}\right)\right\}\left|v_{1}-v^{\prime}\right|^{\gamma+\nu} \frac{\mathrm{d} v_{1} \mathrm{~d} \omega}{\left|v^{\prime}-v\right|^{\nu}}
$$


and

$$
\begin{aligned}
Q_{2}(f, g)(v) \equiv \int_{\mathbb{R}_{v_{1}}^{3}} \int_{S_{\omega}^{2}}\left\{f\left(v^{\prime}\right) g\left(v_{1}^{\prime}\right)-f(v) g\left(v_{1}\right)\right\} & \\
& \times\left[\left\{\left|v_{1}-v^{\prime}\right|^{2}+\left|v^{\prime}-v\right|^{2}\right\}^{\frac{\gamma+\nu}{2}}-\left|v_{1}-v^{\prime}\right|^{\gamma+\nu}\right] \frac{\mathrm{d} v_{1} \mathrm{~d} \omega}{\left|v^{\prime}-v\right|^{\nu}} .
\end{aligned}
$$

Our next task will be to analyse each of these two terms.

Let us begin with $Q_{2}$, and set

$$
B^{c}\left(\left|v-v_{1}\right|,\left|\left(\frac{v-v_{1}}{\left|v-v_{1}\right|}, \omega\right)\right|\right) \equiv \frac{1}{\left|v^{\prime}-v\right|^{\nu}}\left[\left\{\left|v_{1}-v^{\prime}\right|^{2}+\left|v^{\prime}-v\right|^{2}\right\}^{\frac{\gamma+\nu}{2}}-\left|v_{1}-v^{\prime}\right|^{\gamma+\nu}\right] .
$$

$B^{c}$ depends on the same variable as $B$, as $\left\{\left|v_{1}-v^{\prime}\right|^{2}+\left|v^{\prime}-v\right|^{2}\right\}^{\frac{\gamma+\nu}{2}}=\left|v-v_{1}\right|^{\gamma+\nu}$ and

$$
\left|v_{1}-v^{\prime}\right|^{2}=\left|v_{1}-v\right|^{2}\left\{1-\left|\left(\frac{v-v_{1}}{\left|v-v_{1}\right|}, \omega\right)\right|^{2}\right\}
$$

Of course $B^{c} \geq 0$. Next, consider the function

$$
j: t \in \mathbb{R}^{+} \rightarrow\left\{|\alpha|^{2}+t\right\}^{\frac{\gamma+\nu}{2}}
$$

for all $\alpha \in \mathbb{R}^{3}-\{0\}$ fixed. Then

$$
\left|\partial_{t} j\right|=C\left\{|\alpha|^{2}+t\right\}^{\frac{\gamma+\nu}{2}-1} \leq C \frac{1}{|\alpha|^{2-(\gamma+\nu)}},
$$

and as $\frac{\gamma+\nu}{2}=\frac{s-2}{s-1}, 0<\frac{\gamma+\nu}{2}<1$, we deduce that

$$
\left|B^{c}(., .)\right| \leq B_{\max }^{c}(., .) \equiv C \frac{1}{\left|v_{1}-v^{\prime}\right|^{2-(\gamma+\nu)}} \frac{1}{\left|v^{\prime}-v\right|^{\nu-2}} .
$$

Since $(2.5)$

$$
\left|v^{\prime}-v\right|=\left|v_{1}-v\right|\left|\left(\frac{v-v_{1}}{\left|v-v_{1}\right|}, \omega\right)\right| \text { and }\left|v_{1}-v^{\prime}\right|=\left|v_{1}-v\right|\left\{1-\left|\left(\frac{v-v_{1}}{\left|v-v_{1}\right|}, \omega\right)\right|^{2}\right\}^{\frac{1}{2}}
$$

we get

$$
B_{\max }^{c}(., .)=C\left|v_{1}-v\right|^{\gamma} \frac{1}{\left\{1-\left|\left(\frac{v-v_{1}}{\left|v-v_{1}\right|}, \omega\right)\right|^{2}\right\}^{1-\frac{\gamma+\nu}{2}}} \frac{1}{\left|\left(\frac{v-v_{1}}{\left|v-v_{1}\right|}, \omega\right)\right|^{\nu-2}}
$$

Recall that $0<1-\left(\frac{\gamma+\nu}{2}\right)<1,-1<\nu-2<1$ and $-3<\gamma<1$. Note also that $B_{\max }^{c}$ and therefore $B^{c}$ is integrable over $S^{2}$, for fixed $v_{1}-v$. In conclusion, we have shown that $Q_{2}$ given by (2.11) behaves exactly like a Boltzmann operator with a cutoff type kernel, and this is one part of Theorem 2.1.

Next, we consider $Q_{1}$ given by (2.10). We first transform it using the Carleman representation, see [13,34]. 
Lemma 2.1. Assume (2.2a) and (2.3). Then $Q_{1}$ given by (2.10) writes

$$
Q_{1}(f, g)(v)=\int_{\mathbb{R}_{h}^{3}} \frac{2 \mathrm{~d} h}{|h|^{\nu+2}} \int_{E_{0, h}}\{f(v-h) g(\alpha+v)-f(v) g(\alpha+v-h)\}|\alpha|^{\gamma+\nu} .
$$

Proof. We perform the same transformation as used recently by B.Wennberg [34], which transforms the integral over $S^{2}$ of (2.10) into an integral over a plane. In fact, this idea goes back to Carleman [13].

The important difference with [34] is that we keep in the computations the loss term $-f(v) g\left(v_{1}\right)$.

Let us go into the details, referring to [34] for some geometrical insights and for some earlier references.

Let $E_{v, v^{\prime}-v}$ for the plane normal to $v^{\prime}-v$ and containing $v, v^{\prime}$ describing $\mathbb{R}^{3}$. We write $v^{\prime}=q \omega+v$. For fixed $\omega$ and $v$, we may also express $v_{1}$ as $v_{1}=q \omega+v_{1}^{\prime}$, so that $\mathrm{d} v_{1}$ becomes $\mathrm{d} v_{1}^{\prime} \mathrm{d} q$. In the same way, expressing $v^{\prime}$ in polar coordinates with origin at $v, \mathrm{~d} v^{\prime}$ becomes $q^{2} \mathrm{~d} q \mathrm{~d} \omega$ (recall we are in $3 \mathrm{D}$ ). Therefore, we get

$$
\mathrm{d} v_{1} \mathrm{~d} \omega=\mathrm{d} v_{1}^{\prime} \mathrm{d} q \mathrm{~d} \omega=\frac{1}{q^{2}} \mathrm{~d} v_{1}^{\prime} \mathrm{d} v^{\prime},
$$

where $\mathrm{d} v_{1}^{\prime}$ denotes the usual Lebesgue measure on $E_{v, v^{\prime}-v}$ and $\mathrm{d} v^{\prime}$ the Lebesgue measure in $\mathbb{R}^{3}$.

As $v^{\prime}-v=q \omega$, we have also $v_{1}=\left(v^{\prime}-v\right)+v_{1}^{\prime}$, and as $q^{2}=\left|v^{\prime}-v\right|^{2}$, we get also $\mathrm{d} v_{1} \mathrm{~d} \omega=\frac{1}{\left|v^{\prime}-v\right|^{2}} \mathrm{~d} v_{1}^{\prime} \mathrm{d} v^{\prime}$.

Finally, using the relation $\left|v^{\prime}-v_{1}\right|=\left|v_{1}^{\prime}-v\right|$, we obtain the following expression for $Q$

$$
Q(f, g)(v) \equiv \int_{\mathbb{R}_{v^{\prime}}^{3}} \frac{2 \mathrm{~d} v^{\prime}}{\left|v^{\prime}-v\right|^{\nu+2}} \int_{E_{v, v-v^{\prime}}}\left\{f\left(v^{\prime}\right) g\left(v_{1}^{\prime}\right)-f(v) g\left(v^{\prime}-v+v_{1}^{\prime}\right)\right\}\left|v_{1}^{\prime}-v\right|^{\gamma+\nu} \mathrm{d} v_{1}^{\prime} .
$$

For $v^{\prime}$ fixed, we make the change of variables $v_{1}^{\prime} \rightarrow \alpha=v_{1}^{\prime}-v^{\prime}$, that is $v_{1}^{\prime}=\alpha+v^{\prime}$, and we find that it also writes

$$
\int_{\mathbb{R}_{v^{\prime}}^{3}} \frac{2 \mathrm{~d} v^{\prime}}{\left|v^{\prime}-v\right|^{\nu+2}} \int_{E_{v-v^{\prime}, v-v^{\prime}}}\left\{f\left(v^{\prime}\right) g\left(\alpha+v^{\prime}\right)-f(v) g\left(v^{\prime}-v+\alpha+v^{\prime}\right)\right\}\left|\alpha+v^{\prime}-v\right|^{\gamma+\nu} \mathrm{d} \alpha,
$$

then, again $\alpha=\alpha^{\prime}+v-v^{\prime}$, getting (change $\alpha^{\prime}$ to the notation $\alpha$ at the end)

$$
Q(f, g)(v) \equiv \int_{\mathbb{R}_{v^{\prime}}^{3}} \frac{2 \mathrm{~d} v^{\prime}}{\left|v^{\prime}-v\right|^{\nu+2}} \int_{E_{0, v-v^{\prime}}}\left\{f\left(v^{\prime}\right) g(\alpha+v)-f(v) g\left(\alpha+v^{\prime}\right)\right\}|\alpha|^{\gamma+\nu} \mathrm{d} \alpha,
$$

and making the change in $v^{\prime}, h=v-v^{\prime}$, noticing $(\alpha, h)=0$, we conclude the proof of Lemma 2.1.

Next, we introduce the

Definition 2.1. For $u \in S^{2}, v \in \mathbb{R}^{3}$, let

$$
\beta_{g}(u, v) \equiv \int_{E_{0, u}} g(\alpha+v)|\alpha|^{\gamma+\nu} \mathrm{d} \alpha .
$$

We set $Q_{1}=Q_{1,1}+Q_{1,2}$ where

$$
\begin{aligned}
& Q_{1,1}(f, g)(v)=\int_{\mathbb{R}_{h}^{3}} \frac{2 \mathrm{~d} h}{|h|^{\nu+2}}\{f(v-h)-f(v)\} \beta_{g}\left(\frac{h}{|h|}, v\right), \\
& Q_{1,2}(f, g)(v)=\int_{\mathbb{R}_{h}^{3}} \frac{2 \mathrm{~d} h}{|h|^{\nu+2}}\left\{\beta_{g}\left(\frac{h}{|h|}, v\right)-\beta_{g}\left(\frac{h}{|h|}, v-h\right)\right\} \times f(v) .
\end{aligned}
$$

Now, we can start with $Q_{1,1}$, for which one has 
Lemma 2.2. Let $Q_{1,1}$ be given by Definition 2.1. Then, there exists a fixed constant $C_{s}^{\prime}$ depending only on $s$ (or $\nu)$ such that

$$
Q_{1,1}(f, g)(v)=-C_{s}^{\prime} \int_{\mathbb{R}_{\alpha}^{3}} \mathrm{~d} \alpha g(\alpha+v)|\alpha|^{\gamma+\nu}|S(\alpha) D|^{\nu-1}(f)(v) .
$$

The value of $C_{s}^{\prime}$ may be found below.

Proof. According to Definition 2.1, $\beta_{g}(-u, v)=\beta_{g}(u, v)$, so that making the change of variables $h \rightarrow-h$, one finds

$$
Q_{1,1}(f, g)(v)=\int_{\mathbb{R}_{h}^{3}} \frac{\mathrm{d} h}{|h|^{\nu+2}}\{f(v-h)+f(v+h)-2 f(v)\} \beta_{g}\left(\frac{h}{|h|}, v\right) .
$$

Writing $h$ in polar coordinates, $h=r \omega$, with $r=|h|, \omega=\frac{h}{|h|}$, we get also

$$
Q_{1,1}(f, g)(v)=\int_{0}^{+\infty} \mathrm{d} r \int_{S_{\omega}^{2}} \mathrm{~d} \omega \frac{1}{r^{\nu}}\{f(v+r \omega)+f(v-r \omega)-2 f(v)\} \beta_{g}(\omega, v) .
$$

Next, letting $\hat{f}(\xi)$ denote the Fourier transform of $f,(2.16)$ writes

$$
Q_{1,1}(f, g)(v)=\int_{\mathbb{R}_{\xi}^{3}} \mathrm{~d} \xi \hat{f}(\xi) e^{i \xi \cdot v} \int_{S_{\omega}^{2}} \mathrm{~d} \omega \beta_{g}(\omega, v)\left\{\int_{0}^{+\infty} \frac{\mathrm{d} r}{r^{\nu}}\left(e^{i r \xi \cdot \omega}+e^{-i r \xi \cdot \omega}-2\right)\right\} .
$$

By classical homogeneity arguments, one has

$$
\begin{gathered}
\int_{0}^{\infty} \frac{\mathrm{d} r}{r^{\nu}}\left(e^{i r \xi \cdot \omega}+e^{-i r \xi \cdot \omega}-2\right) \equiv-C_{s}|\xi \cdot \omega|^{\nu-1}, \\
C_{s} \equiv \int_{0}^{\infty} \frac{\mathrm{d} r}{r^{\nu}}\left(2-e^{i r}-e^{-i r}\right) .
\end{gathered}
$$

Turning to (2.17), we have obtained

$$
Q_{1,1}(f, g)(v)=-C_{s} \int_{\mathbb{R}_{\xi}^{3}} \mathrm{~d} \xi \hat{f}(\xi) e^{i \xi \cdot v} \int_{S_{\omega}^{2}} \mathrm{~d} \omega \beta_{g}(\omega, v)|\xi \cdot \omega|^{\nu-1}
$$

Next, again from Definition 2.1, one has

$$
\beta_{g}(\omega, v)=\int_{\mathbb{R}_{\alpha}^{3}} \mathrm{~d} \alpha \delta_{\alpha \cdot \omega=0} g(\alpha+v)|\alpha|^{\gamma+\nu},
$$

according to which (2.20) transforms into

$$
Q_{1,1}(f, g)(v)=-C_{s} \int_{\mathbb{R}_{\xi}^{3}} \mathrm{~d} \xi \hat{f}(\xi) e^{i \xi \cdot v} \int_{S_{\omega}^{2}} \mathrm{~d} \omega \int_{\mathbb{R}_{\alpha}^{3}} \mathrm{~d} \alpha \delta_{\alpha \cdot \omega=0} g(\alpha+v)|\alpha|^{\gamma+\nu}|\xi \cdot \omega|^{\nu-1}
$$


that is also

$$
Q_{1,1}(f, g)(v)=-\int_{\mathbb{R}_{\xi}^{3}} \mathrm{~d} \xi \int_{\mathbb{R}_{\alpha}^{3}} \mathrm{~d} \alpha \hat{f}(\xi) e^{i \xi \cdot v} g(\alpha+v)|\alpha|^{\gamma+\nu}\left\{C_{s} \int_{S_{\omega}^{2}, \omega \cdot \alpha=0} \mathrm{~d} \omega|\xi \cdot \omega|^{\nu-1}\right\}
$$

and again, we define the constant $C_{s}^{\prime}$ as

$$
\left\{C_{s} \int_{S_{\omega}^{2}, \omega \cdot \alpha=0} \mathrm{~d} \omega|\xi \cdot \omega|^{\nu-1}\right\}=C_{s}^{\prime}|S(\alpha) \cdot \xi|^{\nu-1}
$$

Getting back to $(2.22)$, we find

$$
Q_{1,1}(f, g)(v)=-C_{s}^{\prime} \int_{\mathbb{R}_{\xi}^{3}} \mathrm{~d} \xi \int_{\mathbb{R}_{\alpha}^{3}} \mathrm{~d} \alpha|\alpha|^{\gamma+\nu} g(\alpha+v)|S(\alpha) \cdot \xi|^{\nu-1} \hat{f}(\xi) e^{i \xi \cdot v}
$$

which is nothing else than the expression given in the statement of the Lemma.

In the same way, one has the following result concerning $Q_{1,2}$.

Lemma 2.3. Let $Q_{1,2}$ be given by Definition 2.1. Then, with the same constant $C_{s}^{\prime}$ as in Lemma 2.2, one has

$$
Q_{1,2}(f, g)(v)=C_{s}^{\prime} \cdot f(v) \cdot \int_{\mathbb{R}_{\alpha}^{3}} \mathrm{~d} \alpha|\alpha|^{\gamma+\nu}|S(\alpha) \cdot D|^{\nu-1}(g)(\alpha+v)
$$

Proof. As in the proof of Lemma 2.2, we find first

$$
Q_{1,2}(f, g)(v)=f(v) \int_{0}^{+\infty} \mathrm{d} r \int_{S_{\omega}^{2}} \mathrm{~d} \omega \frac{2}{r^{\nu}}\left\{\beta_{g}(\omega, v)-\beta_{g}(\omega, v-r \omega)\right\}
$$

and introducing $\hat{\beta}_{g}(\omega, \xi)$ for the Fourier transform of $\beta_{g}(\omega, v)$ with respect to the variable $v$, we get

$$
Q_{1,2}(f, g)(v)=f(v) \int_{0}^{+\infty} \mathrm{d} r \int_{S_{\omega}^{2}} \mathrm{~d} \omega \int_{\mathbb{R}_{\xi}^{3}} \mathrm{~d} \xi \frac{2}{r^{\nu}} \hat{\beta}_{g}(\omega, \xi) e^{i \xi \cdot v}\left\{1-e^{-i r \xi \cdot \omega}\right\}
$$

Shifting $\omega$ into $-\omega$, one finds also

$$
Q_{1,2}(f, g)(v)=f(v) \int_{S_{\omega}^{2}} \mathrm{~d} \omega \int_{\mathbb{R}_{\xi}^{3}} \mathrm{~d} \xi \hat{\beta}_{g}(\omega, \xi) e^{i \xi \cdot v}\left\{\int_{0}^{+\infty} \frac{\mathrm{d} r}{r^{\nu}}\left(2-e^{-i r \xi \cdot \omega}-e^{i r \xi \cdot \omega}\right)\right\}
$$

The curly brackets term is nothing else than $C_{s}|\xi \cdot \omega|^{\nu-1}$ as given by (2.19) and (2.23), so that

$$
Q_{1,2}(f, g)(v)=+C_{s} \cdot f(v) \cdot \int_{S_{\omega}^{2}} \mathrm{~d} \omega \int_{\mathbb{R}_{\xi}^{3}} \mathrm{~d} \xi \hat{\beta}_{g}(\omega, \xi) e^{i \xi \cdot v}|\xi \cdot \omega|^{\nu-1}
$$


Next, we express $\hat{\beta}_{g}(\omega, \xi)$ in terms of $\beta_{g}$, and we have the following computations, where we use the same trick as in $(2.21)$

$$
\begin{aligned}
Q_{1,2}(f, g)(v) & =+C_{s} \cdot f(v) \cdot \int_{\mathbb{R}_{\xi}^{3}} \mathrm{~d} \xi \int_{S_{\omega}^{2}} \mathrm{~d} \omega \int_{\mathbb{R}_{k}^{3}} \mathrm{~d} k \beta_{g}(\omega, k) e^{-i \xi \cdot k} e^{i \xi \cdot v}|\xi \cdot \omega|^{\nu-1} \\
& =+C_{s} \cdot f(v) \cdot \int_{\mathbb{R}_{\xi}^{3}} \mathrm{~d} \xi \int_{S_{\omega}^{2}} \mathrm{~d} \omega \int_{\mathbb{R}_{k}^{3}} \mathrm{~d} k \int_{\mathbb{R}_{\alpha}^{3}} \mathrm{~d} \alpha g(\alpha+k)|\alpha|^{\gamma+\nu} \delta_{\alpha \cdot \omega=0} e^{-i \xi \cdot k} e^{i \xi \cdot v}|\xi \cdot \omega|^{\nu-1} \\
& =f(v) \cdot \int_{\mathbb{R}_{\xi}^{3}} \mathrm{~d} \xi \int_{\mathbb{R}_{k}^{3}} \mathrm{~d} k \int_{\mathbb{R}_{\alpha}^{3}} \mathrm{~d} \alpha|\alpha|^{\gamma+\nu} g(\alpha+k) e^{-i \xi \cdot k} e^{i \xi \cdot v}\left\{C_{s} \int_{S_{\omega}^{2}, \omega \cdot \alpha=0} \mathrm{~d} \omega|\xi \cdot \omega|^{\nu-1}\right\},
\end{aligned}
$$

which, in view of (2.23), yields

$$
Q_{1,2}(f, g)(v)=C_{s}^{\prime} \cdot f(v) \int_{\mathbb{R}_{\xi}^{3}} \int_{\mathbb{R}_{\alpha}^{3}}|\alpha|^{\gamma+\nu} e^{i \xi \cdot v}|S(\alpha) \cdot \xi|^{\nu-1} \int_{\mathbb{R}_{k}^{3}} \mathrm{~d} k g(\alpha+k) e^{-i \xi \cdot k} \mathrm{~d} k .
$$

Finally

$$
Q_{1,2}(f, g)(v)=+C_{s}^{\prime} \cdot f(v) \cdot \int_{\mathbb{R}_{\alpha}^{3}} \mathrm{~d} \alpha \int_{\mathbb{R}_{\xi}^{3}} \mathrm{~d} \xi|\alpha|^{\gamma+\nu} \hat{g}(\xi) e^{i \xi \cdot(\alpha+v)}|S(\alpha) \cdot \xi|^{\nu-1}
$$

and we recognize the expression settled in the Lemma.

Collecting all the above results, the proof of Theorem 2.1 is ended.

Remark 2.5. We would like to produce a second formulation of $Q$, although we have not found a rigorous functional framework in order to use it.

This formulation is still based on the Carleman transform as used in Lemma 2.1. But this time, we proceed directly from (2.1). Then, one can show that

$$
Q(f, f)(v)=\int_{\mathbb{R}_{\alpha}^{3}} \mathrm{~d} \alpha \int_{\mathbb{R}_{h}^{3}} \frac{2 \mathrm{~d} h}{|h|^{\nu+2}} \delta_{\alpha . h=0}\{f(v-h) f(\alpha+v)-f(v) f(\alpha+v-h)\} \times|\alpha+h|^{\gamma+\nu} \mathrm{d} \alpha .
$$

In this integral, for fixed $v$, we make the change of variables in $\alpha$ as $\alpha+v=w$, that is $\alpha=w-v$, getting

$$
Q(f, f)(v)=\int_{\mathbb{R}_{w}^{3}} \int_{\mathbb{R}_{h}^{3}} \frac{2 \mathrm{~d} h}{|h|^{\nu+2}} \delta_{(v-w) . h=0}\{f(v-h) f(w)-f(v) f(w-h)\} \times|v-w+h|^{\gamma+\nu} \mathrm{d} w .
$$

Noticing $|v-w+h|^{\gamma+\nu}=|v-w-h|^{\gamma+\nu}$ as $(v-w) . h=0$, we split (2.30) as

$$
Q(f, f)(v)=Q_{1}(f, f)(v)+Q_{2}(f, f)(v),
$$

where

$$
\left\{\begin{array}{l}
Q_{1}(f, f)(v)=\int_{\mathbb{R}_{h}^{3}} \mathrm{~d} h \frac{2 \mathrm{~d} h}{|h|^{\nu+2}} \delta_{(v-w) \cdot h=0} \times \\
\left\{f(v-h) f(w)|v-w-h|^{\gamma+\nu}-f(v) f(w)|v-w|^{\gamma+\nu}\right\}
\end{array}\right.
$$

and

$$
\left\{\begin{array}{l}
Q_{2}(f, f)(v)=\int_{\mathbb{R}_{w}^{3}} \mathrm{~d} w \int_{\mathbb{R}_{h}^{3}} \mathrm{~d} h \frac{2 \mathrm{~d} h}{|h|^{\nu+2}} \delta_{(v-w) \cdot h=0} \times \\
\left\{f(v) f(w)|v-w|^{\gamma+\nu}-f(v) f(w-h)|v-w+h|^{\gamma+\nu}\right\} .
\end{array}\right.
$$


Now, note that in (2.31), we have a difference operator of step $h$ applied to the function of $v: v \rightarrow f(v) f(w) \mid$ $v-\left.w\right|^{\gamma+\nu}$ while in (2.32) it applies to the function of $w: w \rightarrow f(v) f(w)|v-w|^{\gamma+\nu}$, with $w$ and $v$ respectively fixed.

Then using the ideas of proofs of Lemma 2.2 and Lemma 2.3, one obtains

$$
\begin{aligned}
Q(f, g)=-C_{s}^{\prime} \int_{\mathbb{R}_{w}^{3}} \mathrm{~d} w\left|S(v-w) \cdot D_{v}\right|^{\nu-1}\left(f(v) g(w)|v-w|^{\gamma+\nu}\right) & \\
& +C_{s}^{\prime} \int_{\mathbb{R}_{w}^{3}} \mathrm{~d} w\left|S(v-w) \cdot D_{w}\right|^{\nu-1}\left(f(v) g(w)|v-w|^{\gamma+\nu}\right),
\end{aligned}
$$

with the same constant $C_{s}^{\prime}$ as in Theorem 2.1 .

Note that this second formulation seems easier to handle than the previous one, but it turns out that the mathematical analysis appears to be more involved. In particular, the pdo acts also on the weight function, while in the formulation given in Theorem 2.1, this one was part of the symbol. Yet, one advantage over the first one is that we can consider more general kernels $B$ (see Remark 2.3). Here is one important example. Assume that $B$ is given by

$$
B\left(\left|v-v_{1}\right|,\left|\left(\frac{v-v_{1}}{\left|v-v_{1}\right|}, \omega\right)\right|\right) \equiv \chi\left(\left|v-v_{1}\right|\right)\left|v-v_{1}\right|^{\gamma} \frac{1}{\left|\left(\frac{v-v_{1}}{\left|v-v_{1}\right|}, \omega\right)\right|^{\nu}}
$$

where $\chi$ is a given positive (say smooth) function. Then (2.33) holds true, provided the moment $|v-w|^{\gamma+\nu}$ is turned into $|v-w|^{\gamma+\nu} \chi(|v-w|)$.

\section{Coulomb Decomposition}

In the following, let again $f$ be a smooth function. The Boltzmann Coulomb operator is given (essentially) by

$$
Q(f)(v)=\int_{\mathbb{R}_{v_{1}}^{3}} \int_{S_{\omega}^{2}} 1_{\left|\left(\frac{v-v_{1}}{\left|v-v_{1}\right|}, \omega\right)\right| \geq \delta}\left\{f\left(v^{\prime}\right) f\left(v_{1}^{\prime}\right)-f(v) f\left(v_{1}\right)\right\}\left|v-v_{1}\right|^{-3} \frac{1}{\left|\left(\frac{v-v_{1}}{\left|v-v_{1}\right|}, \omega\right)\right|^{3}} .
$$

Above, $\delta>0$ and $\delta<<1$ is a fixed constant.

The main result of this section is given by

Theorem 3.1. The Boltzmann-Coulomb Q given by (3.1) may be written as

$$
Q(f)(v)=Q_{1}(f)(v)+Q_{2}(f)(v)
$$

with

$$
\begin{aligned}
Q_{1}(f)(v) & =-a\left(v, D_{v}\right)(f)(v), a(v, \xi)=\int_{\alpha} f(\alpha+v) \frac{1}{|\alpha|^{2}} \chi(|\alpha \wedge \xi|), \\
Q_{2}(f)(v) & =f(v) \int_{\alpha} \int_{\xi} \frac{1}{|\alpha|^{2}} \chi(|\alpha \wedge \xi|) \hat{f}(\xi) e^{i \xi \cdot(\alpha+v)}, \chi(t)=S_{\mu}(t)+\bar{S}_{\mu}(t), \\
S_{\mu}(t) & =\frac{C}{3} \int_{\mu t}^{+\infty} \mathrm{d} r \frac{\sin ^{2} r}{r^{3}}, \bar{S}_{\mu}(t)=\frac{C}{3} \frac{1}{\mu^{3} t} \int_{0}^{\mu t} \sin ^{2} r \mathrm{~d} r
\end{aligned}
$$

where $C$ is a fixed $>0$ constant, $\mu=\frac{\delta}{\sqrt{1-\delta^{2}}}$ and $t>0$. 
Remark 3.1. The above result is only but a preliminary step in the construction and study of associated solutions. For instance, it should provide at least small solutions, as shown in the non cutoff case in [6]. Note that a weak formulation is already sufficient, see [9], but, and again as in Section 2, we expect that it will prove useful, at least for regularity questions or in a linearised context.

Remark 3.2. One has the following estimates

$$
\left|S_{\mu}(|\alpha \wedge \xi|)\right|+\left|\bar{S}_{\mu}(|\alpha \wedge \xi|)\right| \leq \frac{C}{\mu^{2}},\left|\bar{S}_{\mu}(|\alpha \wedge \xi|)\right| \leq C|\alpha \wedge \xi|^{2} .
$$

Proof. (of Theorem 3.1).

Firstly, we make use of the Carleman's representation as in Section 2 to get

$$
Q(f)(v)=\int_{\mathbb{R}_{v_{1}}^{3}} \int_{S_{\omega}^{2}, \frac{\left|v^{\prime}-v\right|}{\left|v_{1}-v\right|} \geq \delta}\left\{f\left(v^{\prime}\right) f\left(v_{1}^{\prime}\right)-f(v) f\left(v_{1}\right)\right\} \frac{\mathrm{d} v_{1} \mathrm{~d} \omega}{\left|v^{\prime}-v\right|^{3}},
$$

as $\left|v_{1}-v\right|=\left|v_{1}^{\prime}-v^{\prime}\right|$ and then

$$
Q(f)(v)=\int_{\mathbb{R}_{v^{\prime}}^{3}} \frac{2 \mathrm{~d} v^{\prime}}{\left|v^{\prime}-v\right|^{5}} \int_{E_{v, v-v^{\prime}}}\left\{f\left(v^{\prime}\right) f\left(v_{1}^{\prime}\right)-f(v) f\left(v^{\prime}-v+v_{1}^{\prime}\right)\right\} 1_{\frac{\left|v^{\prime}-v\right|}{\left|v_{1}^{\prime}-v^{\prime}\right|} \geq \delta} .
$$

Here and below, recall that $E_{\alpha, \beta}$ denotes the hyperplan passing through $\alpha$ and orthogonal to $\beta$.

For $v^{\prime}$ fixed, make the change $\alpha=v_{1}^{\prime}-v^{\prime}$ to obtain

$$
Q(f)(v)=\int_{\mathbb{R}_{v^{\prime}}^{3}} \frac{2 \mathrm{~d} v^{\prime}}{\left|v^{\prime}-v\right|^{5}} \int_{E_{v-v^{\prime}, v-v^{\prime}}}\left\{f\left(v^{\prime}\right) f\left(\alpha+v^{\prime}\right)-f(v) f\left(v^{\prime}-v+\alpha+v^{\prime}\right)\right\} 1_{\frac{\left|v^{\prime}-v\right|}{|\alpha|} \geq \delta}
$$

then, for fixed $v^{\prime}$ make the change $\alpha^{\prime}=\alpha-v+v^{\prime}$ and then the change for $v^{\prime}$ fixed, $h=v-v^{\prime}$ to obtain finally

$$
Q(f)(v)=\int_{\mathbb{R}_{h}^{3}} \frac{2 \mathrm{~d} h}{|h|^{5}} \int_{E_{0, h}}\{f(v-h) f(\alpha+v)-f(v) f(\alpha+v-h)\} 1_{\mid \frac{|h|}{|\alpha-h|} \geq \delta} .
$$

We split $Q$ according to

$$
Q(f)=Q_{1}(f)+Q_{2}(f)
$$

where

$$
\begin{gathered}
Q_{1}(f)=\int_{\mathbb{R}_{h}^{3}} \frac{2 \mathrm{~d} h}{|h|^{5}} \int_{E_{0, h}} f(\alpha+v)\{f(v-h)-f(v)\} 1_{\frac{|h|}{|\alpha-h|} \geq \delta}, \\
Q_{2}(f)(v)=f(v) \int_{\mathbb{R}_{h}^{3}} \frac{2 \mathrm{~d} h}{|h|^{5}} \int_{E_{0, h}}\{f(\alpha+v)-f(\alpha+v-h)\} 1_{\frac{|h|}{|\alpha-h|} \geq \delta} .
\end{gathered}
$$

Let us first study $Q_{1}$. Proceeding as in Section 2,

$$
Q_{1}(f)(v)=\int_{\xi} \hat{f}(\xi) e^{i \xi \cdot v} \operatorname{Symb}(v, \xi),
$$


where

$$
\begin{aligned}
\operatorname{Symb}(v, \xi) & =\int_{\mathbb{R}_{h}^{3}} \frac{\mathrm{d} h}{|h|^{5}} \int_{E_{0, h}} f(\alpha+v)\{1-\cos (h \cdot \xi)\} 1_{\frac{|h|^{2}}{|\alpha|^{2}+|h|^{2}} \geq \delta^{2}} \\
& =\int_{0}^{\infty} \int_{S_{\omega}^{2}} \frac{\mathrm{d} r}{r^{3}} \int_{E_{0, \omega}} \mathrm{d} \alpha f(\alpha+v)\{1-\cos (r \omega \cdot \xi)\} 1_{\frac{r^{2}}{|\alpha|^{2}+r^{2}} \geq \delta^{2}} \\
& =\int_{\mathbb{R}_{\alpha}^{3}} f(\alpha+v) \int_{S_{\omega}^{2}, \omega \cdot \alpha=0} \mathrm{~d} \omega \int_{0}^{\infty} \mathrm{d} r \frac{1-\cos (r \omega \cdot \xi)}{r^{3}} 1_{\frac{r^{2}}{|\alpha|^{2}+r^{2}} \geq \delta^{2}} .
\end{aligned}
$$

Next, note that, as $\delta>0$ and $\delta<<1$, one has denoting $\mu=\frac{\delta}{\sqrt{1-\delta^{2}}}>0, \frac{r^{2}}{|\alpha|^{2}+r^{2}} \geq \delta^{2}$ if and only if $r \geq \mu|\alpha|$. Therefore, one obtains

$$
\operatorname{Symb}=C \int_{\mathbb{R}_{\alpha}^{3}} f(\alpha+v) \int_{S_{\omega}^{2}, \omega \cdot \alpha=0} \mathrm{~d} \omega \int_{\mu|\alpha|}^{\infty} \frac{\sin ^{2}(r|\omega \cdot \xi|)}{r^{3}} \mathrm{~d} r
$$

Let us set

$$
\mathbf{a} \equiv \int_{S_{\omega}^{2}, \omega \cdot \alpha=0} \mathrm{~d} \omega \int_{\mu|\alpha|}^{\infty} \frac{\sin ^{2}(r|\omega \cdot \xi|)}{r^{3}} \mathrm{~d} r .
$$

Actually, note that the sole difficulty arises when $\alpha=0$. We now study a. One has first, making the change of variable $r^{\prime}=r|\omega \cdot \xi|$ that

$$
\mathbf{a}=\int_{S_{\omega}^{2}, \omega \cdot \alpha=0} \mathrm{~d} \omega \int_{\mu|\alpha||\omega \cdot \xi|}^{\infty}|\omega \cdot \xi|^{2} \frac{\sin ^{2} r}{r^{3}} \mathrm{~d} r=\frac{1}{|\alpha|^{2}}\left\{\int_{\mu|\alpha||\omega . \xi|}^{\infty}|| \alpha|\omega \cdot \xi|^{2} \frac{\sin ^{2} r}{r^{3}} \mathrm{~d} r\right\} .
$$

Note the brackets term above is of the form $\psi_{\mu}(|\alpha \wedge \xi|)$. Actually, we can push the computations a little further. Start from

$$
\begin{aligned}
\mathbf{a} & =\int_{S_{\omega}^{2}, \omega \cdot \alpha=0} \mathrm{~d} \omega \int_{0}^{\infty}|\omega \cdot \xi|^{2} \frac{\sin ^{2} r}{r^{3}} 1_{r \geq \mu|\alpha||\omega \cdot \xi|} \mathrm{d} r \\
& =\int_{0}^{\infty} \mathrm{d} r \frac{\sin ^{2} r}{r^{3}} \int_{S_{\omega}^{2}, \omega \cdot \alpha=0} \mathrm{~d} \omega|\omega \cdot \xi|^{2} 1_{|\omega \cdot \xi| \leq \frac{r}{\mu|\alpha|}} \\
& =\int_{0}^{\infty} \mathrm{d} r \frac{\sin ^{2} r}{r^{3}} \int_{S_{\omega}^{2}, \omega \cdot \alpha=0} \mathrm{~d} \omega|\omega \cdot S(\alpha) \xi|^{2} 1_{|\omega \cdot S(\alpha) \xi| \leq \frac{r}{\mu|\alpha|}}
\end{aligned}
$$

Therefore

$$
\mathbf{a}=|S(\alpha) \xi|^{2} \int_{0}^{\infty} \mathrm{d} r \frac{\sin ^{2} r}{r^{3}} \mathbf{b}
$$

with

$$
\mathbf{b}=\int_{S_{\omega}^{2}, \omega \cdot \alpha=0} \mathrm{~d} \omega\left|\omega \cdot \frac{S(\alpha) \xi}{|S(\alpha) \xi|}\right|^{2} 1_{\left|\omega \cdot \frac{S(\alpha) \xi}{|S(\alpha) \xi|}\right| \leq \frac{r}{\mu|\alpha||S(\alpha) \xi|}} .
$$

Shifting into the usual polar coordinates, one has also

$$
\mathbf{b}=\int_{0}^{\frac{\pi}{2}} \cos ^{2} \theta \sin \theta 1_{\cos \theta \leq \frac{r}{\mu|\alpha||S(\alpha) . \xi|}} \mathrm{d} \theta .
$$


If $\frac{r}{\mu|\alpha \wedge \xi|} \geq 1$, so that $r \geq \mu|\alpha \wedge \xi|$, then

$$
\mathbf{b}=\int_{0}^{\frac{\pi}{2}} \cos ^{2} \theta \sin \theta \mathrm{d} \theta=\frac{1}{3}
$$

Else, if $\frac{r}{\mu|\alpha \wedge \xi|}<1$, so that $r<\mu|\alpha \wedge \xi|$, then

$$
\begin{aligned}
\mathbf{b} & =\int_{0}^{\frac{\pi}{2}} \cos ^{2} \theta \sin \theta 1_{\theta \geq \operatorname{Arcos}\left(\frac{r}{\mu|\alpha \wedge \xi|}\right)} \\
& =\int_{\operatorname{Arcos}\left(\frac{r}{\mu|\alpha \wedge \xi|}\right)}^{\frac{\pi}{2}} \cos ^{2} \theta \sin \theta \mathrm{d} \theta \\
& =\frac{1}{3} \frac{r^{3}}{\mu^{3}|\alpha \wedge \xi|^{3}} .
\end{aligned}
$$

Finally, we obtain (if $|\alpha \wedge \xi|=0$ then $\mathbf{b}=0$ )

$$
\mathbf{b}=\frac{1}{3} 1_{r \geq \mu|\alpha \wedge \xi|}+\frac{1}{3} \frac{r^{3}}{\mu^{3}|\alpha \wedge \xi|^{3}} 1_{r<\mu|\alpha \wedge \xi|} .
$$

Getting back to (3.9), we have finally obtained

$$
\begin{aligned}
\mathbf{a} & =|S(\alpha) \xi|^{2} \int_{0}^{\infty} \mathrm{d} r \frac{\sin ^{2} r}{r^{3}} \mathbf{b} \\
& =|S(\alpha) \xi|^{2} \int_{0}^{\infty} \mathrm{d} r \frac{\sin ^{2} r}{r^{3}}\left\{\frac{1}{3} 1_{r \geq \mu|\alpha \wedge \xi|}+\frac{1}{3} \frac{r^{3}}{\mu^{3}|\alpha \wedge \xi|^{3}} 1_{r<\mu|\alpha \wedge \xi|}\right\} \\
& =\frac{1}{|\alpha|^{2}}|\alpha \wedge \xi|^{2}\left[\frac{1}{3} 1_{r \geq \mu|\alpha \wedge \xi|}+\frac{1}{3} \frac{r^{3}}{\mu^{3}|\alpha \wedge \xi|^{3}} 1_{r<\mu|\alpha \wedge \xi|}\right] .
\end{aligned}
$$

Next, set

$$
S_{\mu}(|\alpha \wedge \xi|) \equiv \frac{1}{3}|\alpha \wedge \xi|^{2} \int_{\mu|\alpha \wedge \xi|}^{\infty} \mathrm{d} r \frac{\sin ^{2} r}{r^{3}}
$$

and

$$
\bar{S}_{\mu}(|\alpha \wedge \xi|) \equiv \frac{1}{3} \frac{|\alpha \wedge \xi|^{2}}{\mu^{3}|\alpha \wedge \xi|^{3}} \int_{0}^{\mu|\alpha \wedge \xi|} \sin ^{2} r \mathrm{~d} r
$$

Then

$$
\mathbf{a}=\frac{1}{|\alpha|^{2}} S_{\mu}(|\alpha \wedge \xi|)+\frac{1}{|\alpha|^{2}} \bar{S}_{\mu}(|\alpha \wedge \xi|)
$$

In conclusion, in view of (3.7), the symbol is given by

$$
\operatorname{Symb}(v, \xi) \equiv C \int_{\mathbb{R}_{\alpha}^{3}} f(\alpha+v) \frac{1}{|\alpha|^{2}}\left\{S_{\mu}(|\alpha \wedge \xi|)+\bar{S}_{\mu}(|\alpha \wedge \xi|)\right\}
$$


There remains to analyse $Q_{2}$ as given by (3.5), and in view of the preceding steps and Section 2, it writes as

$$
Q_{2}(f)(v)=f(v) C \int_{\mathbb{R}_{\alpha}^{3}} \int_{\xi} \frac{1}{|\alpha|^{2}}\left\{S_{\mu}(|\alpha \wedge \xi|)+\bar{S}_{\mu}(|\alpha \wedge \xi|)\right\} \hat{f}(\xi) e^{i \xi \cdot(\alpha+v)} \mathrm{d} \xi
$$

This ends up the proof of Theorem 3.1 .

Acknowledgements. I would like to thank Kamal Hamdache, from the beginning of my studies. He suggested some questions, and also quite without purpose, puts me on the (right?) way.

\section{REFERENCES}

[1] R. Alexandre, Sur l'opérateur de Boltzmann linéaire 3D sans troncature angulaire. Note C.R. Acad. Sci. Paris Sér. I 325 (1997) 959-962.

[2] R. Alexandre, Remarks on 3D Boltzmann linear equation without cutoff. Trans. Theory and Stat. Phys. 28 (1999) $433-473$.

[3] R. Alexandre, Sur l'opérateur de Boltzmann non linéaire 3D sans troncature angulaire. Note C.R. Acad. Sci. Paris Sér. I 326 (1998) $165-168$.

[4] R. Alexandre, Sur le taux de dissipation d'entropie sans troncature angulaire. Note C.R. Acad. Sci. Paris Sér. I (1998) 311-315.

[5] R. Alexandre, Une définition des solutions renormalisées pour l'équation de Boltzmann. Note C.R. Acad. Sci. Paris Sér. I 328 (1999) 987-991.

[6] R. Alexandre, The linearised Boltzmann operator and applications. In preparation.

[7] R. Alexandre, Solutions Maxwelliennes pour l'équation de Boltzmann sans troncature angulaire. Note submitted to C.R. Acad. Sci. Paris Sér. I (to appear).

[8] R. Alexandre, L. Desvillettes, C. Villani and B. Wennberg, Entropy dissipation and long range interactions. Arch. Rat. Mech. Anal. (to appear).

[9] R. Alexandre, C. Villani, On the Boltzmann equation for long-range interactions and the Landau approximation in plasma physics. (Preprints ENS Ulm DMA-99-22, 1999).

[10] L. Arkeryd, On the Boltzmann equation. Arch. Rat. Mech. Anal. 45 (1972) 1-34.

[11] L. Arkeryd, Intermolecular forces of infinite range and the Boltzmann equation. Arch. Rat. Mech. Anal. 77 (1981) 11-21.

[12] R. Balescu, Statistical Mechanics of charged particles. Wiley Interscience, N.Y, USA (1963).

[13] T. Carleman, Problèmes Mathématiques dans la Théorie cinétique des Gaz. Almquist and Wiksell, Uppsala (1957)

[14] C. Cercignani, Mathematical Methods in Kinetic Theory. 2nd Ed. Plenum (1990).

[15] C. Cercignani, R. Illner and M. Pulvirenti, The Mathematical Theory of Dilute Gases. Series in Appl. Sci. 106, Springer Verlag, New York (1994).

[16] P. Degond and B. Lucquin, The Fokker-Planck asymptotics of the Boltzmann collision operator in the Coulomb case. Math. Models Methods Appl. Sci. 2-2 (1992) 167-182.

[17] L. Desvillettes, Regularisation properties of the 2D homogeneous Boltzmann equation. Transport Theory Statist. Phys. 26 (1997) 341-357.

[18] L. Desvillettes, Regularisation for the non-cutoff 2D radially symmetric Boltzmann equation. Transport Theory Statist. Phys. 25 (1996) 383-394.

[19] L. Desvillettes, On asymptotics of the Boltzmann equation when the collisions become grazing. Transport Theory Stat. Phys. 21 (1992) 259-276.

[20] L. Desvillettes and B. Wennberg, work in preparation.

[21] R.J. DiPerna and P.L. Lions, On the Cauchy problem for Boltzmann equation; Global existence and weak stability. Ann. Maths. 130 (1989) 321-366.

[22] R.J. DiPerna and P.L. Lions, Global weak solutions of kinetic equations. Sem. Mat. Torino 46 (1988) $259-288$.

[23] T. Goudon, On Boltzmann equations and Fokker-Planck asymptotics. J. Stat. Phys. 89 (1997) $751-776$.

[24] P.L. Lions, Compactness in Boltzmann's equation, via FIO and applic. J. Math. Kyoto Univ. 34 (1994) Part I 391-427; Part II 429-461, Part III 539-584.

[25] P.L. Lions, On Boltzmann and Landau equations. Phil. Trans. Roy. Soc. London A-346 (1994) 191-204.

[26] P.L. Lions, Regularity and compactness for Boltzmann collision operators without angular cutoff. Note C.R. Acad. Sci. Paris Sér. I 326 (1998) 37-41.

[27] Y.P. Pao, Boltzmann Collision Operator with Inverse power Intermolecular potentials. C.P.A.M 27 ( 1974) Part I 407-428; Part II 559-581.

[28] M.E. Taylor, Pseudo-Differential Operators. Princeton Univ. Press (1981).

[29] M.E. Taylor, Pdo and non linear PDE, Birkhauser, Boston (1991).

[30] C. Villani, Contributions à l'étude mathématique des équations de Boltzmann et de Landau en théorie cinétique des gaz et des plasma, Thèse Université Paris-Dauphine (1998). 
[31] C. Villani, Regularity estimates via the entropy dissipation for the spatially homogeneous Boltzmann equation without cut-off. Rev. Mat. Iberoam. (to appear).

[32] C. Villani, Conservative forms of Boltzmann's collision operator: Landau revisited. Math. Mod. Num. An. (1998).

[33] S. Ukai, Solutions of the Boltzmann equation. In: Patterns and Waves, North-Holland (1985).

[34] B. Wennberg, Regularity in the Boltzmann equation and the Radon transform. CPDE 19, (1994) 2057-2074. 